\title{
INVARIANTS OF KNOT DIAGRAMS AND RELATIONS AMONG REIDEMEISTER MOVES
}

\author{
OLOF-PETTER ÖSTLUND
}

\begin{abstract}
In this paper a classification of Reidemeister moves, which is the most refined, is introduced. In particular, this classification distinguishes some $\Omega_{3}$-moves that only differ in how the three strands that are involved in the move are ordered on the knot.

To transform knot diagrams of isotopic knots into each other one must in general use $\Omega_{3}$-moves of at least two different classes. To show this, knot diagram invariants that jump only under $\Omega_{3}$-moves are introduced.

Knot diagrams of isotopic knots can be connected by a sequence of Reidemeister moves of only six, out of the total of 24, classes. This result can be applied in knot theory to simplify proofs of invariance of diagrammatical knot invariants. In particular, a criterion for a function on Gauss diagrams to define a knot invariant is presented.
\end{abstract}

\section{Knot diagrams and Reidemeister moves.}

1.1. Knot diagrams. A knot diagram is a picture of an oriented smooth knot like the figure eight knot diagram in Figure 1. Formally, this is the image of an immersion of $S^{1}$ in $\mathbb{R}^{2}$, with transversal double points and no points of higher multiplicity, which has been decorated so that we can distinguish:

a: An orientation of the strand, and

b: an overpassing and an underpassing strand at each double point.

Of course, the manner of decoration, such as where an arrow that indicates the orientation is placed, does not matter: Two knot diagrams are the same if they are made from the same image, have the same orientation, and have the same over-undercrossing information at each crossing point.

Two knot diagrams are equivalent if there is an orientation-preserving diffeomorphism of the plane that takes one diagram to the other diagram.

1.2. Reidemeister moves. A Reidemeister move is a transformation of a knot diagram which looks like one of the transformations in Table 1 inside a disk in $\mathbb{R}^{2}$ (up to diffeomorphisms) and leaves the knot diagram unchanged outside the disk. More formally, we say that the knot diagrams $k$ and $l$ are related by a Reidemeister

Figure 1. The figure eight knot diagram.

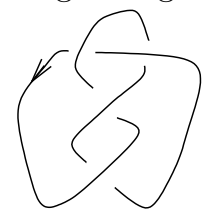


TABLE 1. Reidemeister moves.

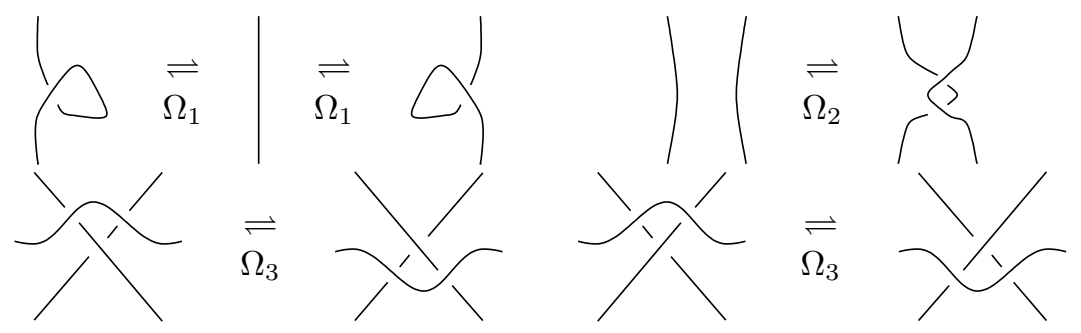

move if they are the same outside $C \subset \mathbb{R}^{2}$ and there are diffeomorphisms $g, h$ from $C$ to a disk $D$ such that

a: $h^{-1} \circ g=I d$ on the boundary of $C$, and

b: $g(k \cap C)$ and $h(l \cap C)$ is one of the unordered pairs of diagram-parts in Table 1 .

We shall call $C$ the changing disk of the Reidemeister move.

Reidemeister moves were introduced in the setting of PL-knots by K. Reidemeister [3] in 1927. Reidemeister proved the PL version of the following well-known fact: Two knot diagrams describe isotopic knots if and only if they are connected by a sequence of Reidemeister moves and orientation-preserving diffeomorphisms of the plane.

1.3. Classifying Reidemeister moves. Reidemeister moves are usually sorted into three classes $\Omega_{1}, \Omega_{2}$ and $\Omega_{3}$, as in Table 1. That is, a move is of class $\Omega_{i}$ if the changing disk contains $i$ strands. (Trivia: In his first paper on the subject [3], Reidemeister put the moves in another order: He called them Operation $1\left(\Omega_{2}\right), 2\left(\Omega_{1}\right)$ and $3\left(\Omega_{3}\right)$. The terminology $\{\Omega .1, \Omega .2, \Omega .3\}$ appears in his book "Knotentheorie" 4 from 1932).

A more refined classification of Reidemeister moves may be needed when one wants to prove that a function defined on knot diagrams is a knot invariant. There are two common ways in which the $\left\{\Omega_{1}, \Omega_{2}, \Omega_{3}\right\}$-classification can be refined: One is to take into account the orientation of $\mathbb{R}^{2}$ (that is, to distinguish the mirrorimaged versions of $\Omega_{1}$ - and $\Omega_{3}$-moves in Table 11). The other is to also take into account the orientations of the strands in the changing disk. In this paper a classification is introduced (see Definition 1 below) which incorporates these refinements and also the ascendingness and descendingness defined in Section 1.4 below. The classification in Definition 1 originated in a study of Gauss diagrams. In Section 2 the classification is reformulated in terms of Gauss diagrams.

1.4. Ascending and descending $\Omega_{3}$-moves. The original part of the classification in Definition 1 is contained in the following characterization of an $\Omega_{3}$-move. We can distinguish the three strands in the changing disk of an $\Omega_{3}$-move: One is the top strand, the strand with two over-crossings. One is the middle strand, and one the bottom strand. When we move along the knot diagram in the direction of the orientation, we pass these strands in some cyclic order. Call the $\Omega_{3}$-move $d e$ scending if we pass the strands in the order top-middle-bottom and call it ascending otherwise. 
Theorem 1. The figure eight knot diagram cannot be transformed into its inverse (the same diagram with the orientation of the strand reversed) without the use of both ascending and descending $\Omega_{3}$-moves. In particular, it cannot be transformed into its inverse without at least two $\Omega_{3}$-moves.

The proof is given in Section 5 .

1.5. A refined classification of Reidemeister moves. By definition, a knot diagram is made from some immersion of $S^{1}$ in $\mathbb{R}^{2}$. We shall assume that the orientation of the knot diagram is induced from the orientation of $S^{1}$, so the knot diagram determines the immersion up to an orientation-preserving diffeomorphism of $S^{1}$.

Let the knot diagrams $d, k$ be decorated images of the immersions $i_{d}, i_{k}: S^{1} \rightarrow$ $\mathbb{R}^{2}$. Given these immersions, an equivalence of the knot diagrams $d$ and $k$ (i.e. an orientation-preserving diffeomorphism of $\mathbb{R}^{2}$ taking $d$ to $k$ ) induces an orientationpreserving diffeomorphism of $S^{1}$.

Definition 1 (Equivalence of Reidemeister moves). Let $X$ be the Reidemeister move that transforms the knot diagram $d_{1}$ to $d_{2}$ inside the changing $\operatorname{disk} C$, and let $Y$ be the Reidemeister move that transforms $k_{1}$ to $k_{2}$ inside the changing disk $D$.

$X$ and $Y$ are directed-equivalent if there are orientation-preserving diffeomorphisms $g, h: C \rightarrow D$ such that

a) $g\left(d_{1} \cap C\right)=k_{1} \cap D$ and $h\left(d_{2} \cap C\right)=k_{2} \cap D$, with the same over-undercrossing information at each crossing.

b) Fix some immersions of the oriented circle such that $d_{1}, d_{2}, k_{1}, k_{2}$ are their decorated images. The maps $g, h$ of pieces of knot diagrams induce maps between segments of the immersing circles. These maps should extend to orientation-preserving diffeomorphisms $S^{1} \rightarrow S^{1}$.

$X$ and $Y$ are equivalent if $X$ is directed-equivalent either to $Y$ or to the reverse move $Y^{-1}$ that changes $k_{2}$ into $k_{1}$ inside $D$.

Every equivalence class splits into exactly two directed equivalence classes; see Section 1.8.

Each crossing in a knot diagram is given a sign by the rule $\operatorname{sign}\left(<\frac{1}{\downarrow}\right)=+1$, $\operatorname{sign}\left(<\frac{1}{1}\right)=-1$

Definition 2 (Taxonomy of Reidemeister moves).

- An $\Omega_{1}$-move is said to be of class $\Omega_{1 i j}, i, j= \pm 1$. The index $i=+1$ if the kink that the move introduces (or removes) is oriented counterclockwise, and $i=-1$ otherwise. The index $j$ is the sign of the crossing that the move introduces or removes.

- An $\Omega_{2}$-move is said to be of class $\Omega_{2 i j}, i, j= \pm 1$. The index $i=+1$ if the meeting strands are oriented in the same direction (the move is called direct), and $i=-1$ otherwise (the move is called inverse). The index $j$ is the sign of the affected crossing where the overcrossing strand is directed out from the area enclosed by the meeting strands:

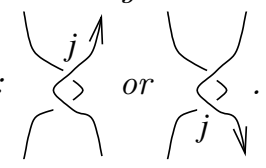


- An $\Omega_{3}$-move is said to be of class $\Omega_{3 i j k m}, i, j, k, m= \pm 1$. The index $m=+1$ if the move is descending and $m=-1$ otherwise. The indices $i, j, k$ are the signs of the crossings in the changing disk:

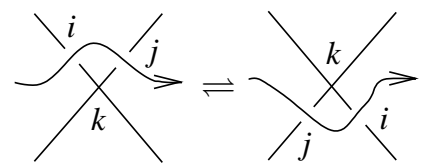

It is easy to verify that Definition 2 gives the splitting of the classes $\Omega_{1}, \Omega_{2}$ and $\Omega_{3}$ into equivalence classes as defined in Definition 1 .

\subsection{Main results on classes of Reidemeister moves.}

Theorem 2. Any Reidemeister move can be realized by a sequence of moves of the classes $\Omega_{1++}, \Omega_{1+-}, \Omega_{2-+}, \Omega_{2--}, \Omega_{3-+++}, \Omega_{3--+-}$, followed by a diffeomorphism with support in the changing disk of the original move.

The proof is given in Section 3. There are other sets of six classes of Reidemeister moves that also has this property, such as the set $\left\{\Omega_{1-+}, \Omega_{1--}, \Omega_{2-+}, \Omega_{2+-}\right.$, $\left.\Omega_{3+--+}, \Omega_{3++--}\right\}$.

Theorem 3. For every knot type there are two knot diagrams, representing knots of this type, such that to transform them into each other we need at least

1. one ascending and one descending $\Omega_{3}$-move, and

2. an $\Omega_{1 i j}$-move and an $\Omega_{1 k l}$-move, $(i, j) \neq(k, l),(i, j) \neq(-k,-l)$.

Theorem 3 extends Theorem 11 to diagrams of any knot type. The proof is given in Section 5, and it makes use of some knot diagram invariants introduced in Section 1 . If we remove requirement 1, the remaining statement can be proved using well-known knot diagram invariants (writhe and winding number, also cf. Proposition 2).

1.7. Organization of the rest of the paper. Sections 1.8 and 1.9 deal with the direction of a move. In Section 2 the definitions and results are reformulated in terms of Gauss diagrams. Section 3 contains the proof of Theorem 2.

In Section 1 several knot diagram invariants are introduced, and some of their properties are derived. Section 4.4 describes how the simplest of the invariants jumps under a Reidemeister move. Section 0 contains the proofs of Theorems 1 and 3 . The proofs make use of some of the invariants from Section 1 .

1.8. Directed classes of Reidemeister moves. Every equivalence class splits into exactly two directed equivalence classes (see Definition 11). We say that an $\Omega_{1}$ - or $\Omega_{2}$-move is in positive direction if the number of crossings in the diagram increases. To define the positive direction of an $\Omega_{3}$-move we use a construction by V. Arnold [1]:

The three strands in the changing disk (before or after the move) form a vanishing triangle. The edges are ordered by the cyclic order in which we pass them if we move around the diagram in the direction of the orientation. This cyclic order gives an orientation of the vanishing triangle. Let $n$ be the number of edges where this orientation coincides with the orientation of the edge. Let $q=(-1)^{n}$. Then $q=-1$ at one side of the move and $q=+1$ on the other. We say that the move is in positive direction if $q=-1$ before the move and $q=+1$ after the move. 
Example 1 (An $\Omega_{3}$-move in positive direction).

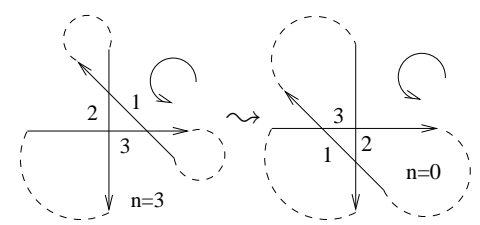

1.9. The direction of ascending and descending $\Omega_{3}$-moves. Arnold's definition of the positive direction of a triple point move appeared in the theory of plane curves. For knot diagrams there is an ordering of the edges in the vanishing triangle which only depends on the knot diagram inside the changing disk: topmiddle-bottom. So it is possible to define a "positive direction" of an $\Omega_{3}$-move in two different ways: Using Arnold's ordering of the edges, or the top-middle-bottom ordering. The two definitions give the same "positive direction" for descending $\Omega_{3}$-moves and opposite "positive direction" for ascending $\Omega_{3}$-moves. (In this paper we always use Arnold's original definition.)

\section{Reidemeister moves of Gauss diagrams.}

Let the knot diagram $d$ be the decorated image of an immersion of an oriented circle, with the orientation of the diagram given by the orientation of the circle. The Gauss diagram $G_{d}$ of $d$ is the immersing oriented circle with the preimages of each crossing point connected by a signed arrow. The arrow points from overpass to underpass and is given the sign of the crossing. Gauss diagrams are considered up to orientation-preserving diffeomorphism of the circle, so equivalent knot diagrams have the same Gauss diagram.

Example 2 (The Gauss diagram of a knot diagram.).

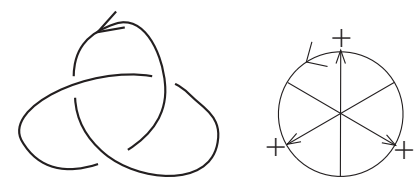

The Gauss diagram determines a knot diagram on the sphere up to an orientationpreserving diffeomorphism of the sphere. That is, the Gauss diagram determines a knot diagram in the plane up to a choice of a point of infinity outside the diagram on the sphere and up to equivalence of knot diagrams.

A Reidemeister move induces a transformation of Gauss diagrams. An $\Omega_{i}$-move affects $i$ arrows, with end points on $i$ segments of the circle of the Gauss diagram, without changing the rest of the diagram.

The original motivation for the choice of the definitions in this paper was the following obvious Proposition:

Proposition 1. The Reidemeister moves $X: d_{1} \mapsto d_{2}$ and $Y: k_{1} \mapsto k_{2}$ are directed-equivalent if and only if the abstract Gauss diagrams obtained by erasing all arrows in $G_{d_{1}}$ and $G_{k_{1}}$ that are not affected by the move are the same (up to an orientation-preserving diffeomorphism of the circle), and likewise for $G_{d_{2}}$ and $G_{k_{2}}$.

That is, $X$ and $Y$ are directed-equivalent if and only if the induced transformations of the Gauss diagrams look the same when disregarding the unchanged part of the diagram. 
TABle 2. A sufficient set of Reidemeister moves for Gauss diagrams.

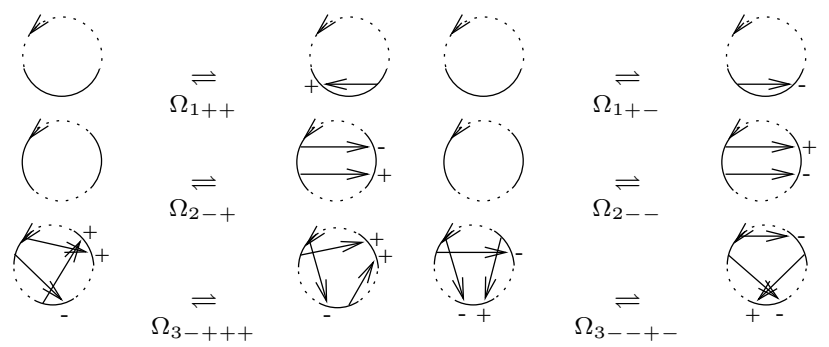

Corollary 1. A function on Gauss diagrams defines a knot invariant if and only if it is invariant under the transformations in Table 6 , where the dotted segments indicate a part of the Gauss diagram that is unchanged.

In Table 2, the positive direction of a move is from left to right.

\section{Proof of Theorem 2.}

The proof of Theorem 2 consists of three lemmas. The method is to draw movies that show explicitly how a Reidemeister move of a class we want to exclude is replaced by a sequence of other Reidemeister moves. All moves in this sequence take place inside the changing disk of the move we replace.

Lemma 1. Any $\Omega_{1}$-move can be replaced by $\Omega_{1++^{-}}, \Omega_{1+-}$ and $\Omega_{2--}-$ moves.

Proof: We can replace an $\Omega_{1-+}$-move with the sequence

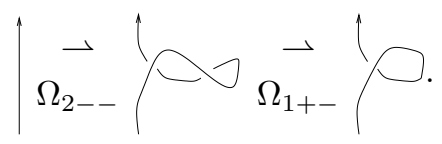

And we can replace an $\Omega_{1--}$-move with the sequence

$$
\mid \begin{gathered}
\rightarrow \\
\Omega_{2--}
\end{gathered} \rightarrow
$$

Lemma 2. Any direct $\Omega_{2}$-move can be replaced by $\Omega_{2--_{--}}, \Omega_{1-+^{-}}, \Omega_{1--_{--}}, \Omega_{3-+++^{-}}$ and $\Omega_{3--+-}$-moves.

Proof: We can replace an $\Omega_{2++}$-move with the sequence

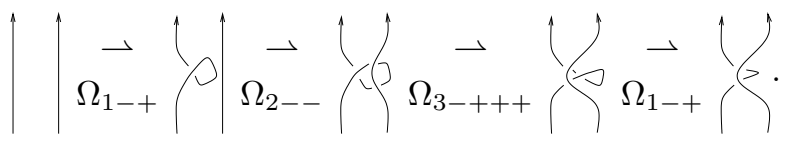

In a similar way we can replace an $\Omega_{2+-}$-move with a sequence of moves of class $\Omega_{1--}, \Omega_{2--}, \Omega_{3--+-}$ and $\Omega_{1--}$.

Lemma 3. Any ascending (descending) $\Omega_{3}$-move can be replaced by $\Omega_{2}$-moves and an $\Omega_{3}$-move of any ascending (descending) class. 
Proof: We can replace an $\Omega_{3}$-move with a sequence which looks like

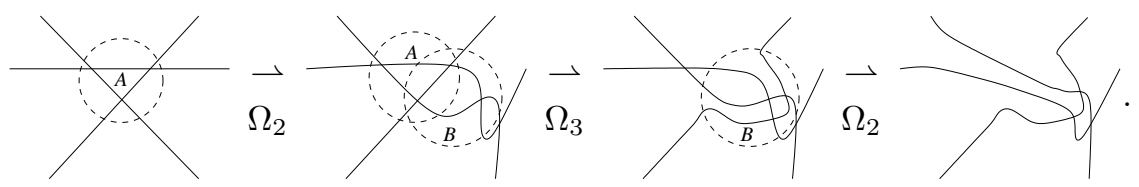

if we temporarily forget about orientation, over/under-crossing information and the ordering of the edges. Here we replace the $\Omega_{3}$-move in the changing disk $A$ with a move in the changing disk $B$. The $\Omega_{3}$-moves in $A$ and in $B$ are either both ascending or both descending. The first three signs $i, j, k$ in the $\Omega_{3 i j k m}$ index are given by the signs of the three crossings in the changing disk as indicated in Definition 2.

We have three choices of wedges where to make the first $\Omega_{2}$-move. If we make the move between the middle and bottom strands, $i$ and $j$ change place in the index and $k$ is reversed: $(i, j, k) \mapsto(j, i,-k)$. If we make the move between the top strand and the strand that crosses the top strand at the $i$-crossing then $(i, j, k) \mapsto(j,-i, k)$. If we make the move between the top strand and the strand that crosses the top strand at the $j$-crossing we get $(i, j, k) \mapsto(-j, i, k)$. Repeating this process with the new changing disk, we can get any index. This completes the proof of Theorem 2.

\section{KNOT DiAgRAm INVARIANTS.}

4.1. Writhe and winding number. Note that by a knot diagram invariant we mean a function on knot diagrams that is unchanged under equivalence of knot diagrams. There are some well-known knot diagram invariants which are not knot invariants, foremost the writhe and the winding number. The writhe is the sum of the signs of all crossings in the diagram. The winding number is the degree of the map $S^{1} \rightarrow S^{1}$ taking a point on the immersing circle to the direction of the tangent vector in the plane.

The main previously known result about classes of Reidemeister moves is Proposition 2 below. Note that the writhe and the winding number are unchanged under $\Omega_{1}$ - and $\Omega_{2}$-moves, which proves the easy "only if"-part of the Proposition.

Proposition 2 (B. Trace [5]). Two knot diagrams of isotopic knots can be transformed into each other without the use of $\Omega_{1}$-moves (they are regularly isotopic) if and only if they have the same writhe and winding number.

4.2. Connected sum of knot diagrams. The knot diagram $k$ is a connected sum of $k_{1}$ and $k_{2}$ if $k$ can be split by a simple closed curve intersecting the diagram in two points, into diagrams equivalent to $k_{1}$ and $k_{2}$, as below:

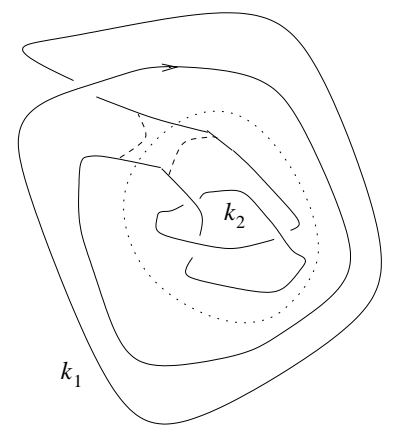


If $k$ is a connected sum of $k_{1}$ and $k_{2}$, the corresponding Gauss diagram $G_{k}$ is a connected sum of $G_{k_{1}}$ and $G_{k_{2}}$ in the sense that $G_{k}$ can be split into $G_{k_{1}}$ and $G_{k_{2}}$ : In the example above, the terms have Gauss diagrams $G_{k_{1}}=$ and $G_{k_{2}}=$ while the sum has Gauss diagram additive if $V(k)=V\left(k_{1}\right)+V\left(k_{2}\right)$ whenever $k$ is a connected sum of $k_{1}$ and $k_{2}$.

Lemma 4. The writhe is additive. The winding number is not, but if $k$ is a connected sum of $k_{1}$ and $k_{2}$, then

$$
\text { winding number }(k)=\text { winding number }\left(k_{1}\right)+\text { winding number }\left(k_{2}\right)+i
$$

where $i$ depends on how the sum is done: $i=+1$ if we cut like $i=-1$ if we cut like

Proof: The first part is evident. For the second part: If we cut the knot diagram $k$ into $k_{1}$ and $k_{2}$ we introduce two new half-turns of the direction vector, in the same direction. Hence the sum of the winding numbers of $k_{1}$ and $k_{2}$ differ by +1 from the winding number of their connected sum $k$ if the half-turns are in positive direction, and by -1 if they are in negative direction.

4.3. The knot diagram invariants $A_{n}, D_{n}$ and $W_{n}$. For a knot diagram $k$ let $A_{4}(k)$ be the sum of the signs of all subdiagrams of the form $a_{4}=$ in the Gauss diagram $G_{k}$ of $k$. A subdiagram is an abstract Gauss diagram that can be created from $G_{k}$ by removing arrows. The sign of the subdiagram is the product of the signs of the arrows in the subdiagram. In the same way define $D_{4}$ as the sum of the signs of the subdiagrams of the form $d_{4}=$. In Section 5 we use $A_{4}$ and $D_{4}$ to prove Theorems 1 and 3 .

More generally, we define $A_{n}(k)$ and $D_{n}(k), n \geq 4$, as the sum of the signs of the subdiagrams of $G_{k}$ with $n$ arrows arranged cyclically as

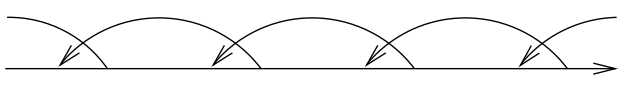

respectively

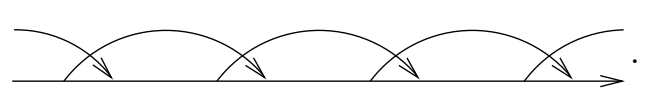

Define $W_{n}(k), n=3,5,7, \ldots$, as the sum of the signs of the subdiagrams of $G_{k}$ with $n$ arrows which are diameters directed so that no two arrow heads are adjacent on the circle. That is, $W_{5}$ is the sum of the signs of the subdiagrams that look like $w_{5}=1$.

Obviously, $A_{n}, D_{n}$ and $W_{n}$ are knot diagram invariants. The abstract subdiagrams that define these invariants, which we call $a_{n}, d_{n}$ and $w_{n}$, are examples of arrow diagrams. Functions defined in this way were introduced by M. Polyak and O. Viro [2]. 
Proposition 3 (Properties of $A_{n}, D_{n}$ and $\left.W_{n}\right) . A_{n}, D_{n}$ and $W_{n}$ are additive under the connected sum of knot diagrams. $A_{n}(k)$ and $D_{n}(k)$ are multiplied by $(-1)^{n}$ by mirroring of $k$ (change of orientation of $\mathbb{R}^{2}$ ) and change into each other under inversion of $k$ (change of orientation of the strand). $W_{n}(k)$ is multiplied by -1 by mirroring of $k$ and is unchanged by inversion of $k$.

Proof: Mirroring of the knot diagram affects the Gauss diagram by reversing the signs of all arrows. The sign of an $a_{n^{-}}, d_{n^{-}}$or $w_{n}$-subdiagram is a product of the signs of $n$ arrows, so every sign is multiplied by $(-1)^{n}$. For a $w_{n}$-subdiagram, $n$ is always odd.

Inversion of the knot diagram affects the Gauss diagram by reversing the orientation of the circle, so the statement is evident.

Additivity is because the Gauss diagram of a connected sum is a connected sum of Gauss diagrams (see Section 4.2), and each $a_{n^{-}}, d_{n^{-}}$, or $w_{n}$-subdiagram evidently must belong to one of the summands.

Proposition 4 (Reidemeister move invariance of $A_{n}, D_{n}$ and $W_{n}$ ). $A_{n}, D_{n}$ and $W_{n}$ do not change under $\Omega_{1}$ - and $\Omega_{2}$-moves. $A_{n}$ does not change under descending $\Omega_{3}$ moves and $D_{n}$ does not change under ascending $\Omega_{3}$-moves.

We assert that $A_{n}\left(D_{n}\right)$ does change under some ascending (descending) $\Omega_{3^{-}}$ moves and $W_{n}$ does change under some $\Omega_{3}$-moves of both ascending and descending type. (That is, they are not knot invariants.)

Proof of $\Omega_{1}$-invariance: An $\Omega_{1}$-move introduces (or removes) one arrow in the Gauss diagram as in Table 2. This arrow is isolated; its head and tail are adjacent on the circle. $a_{n^{-}}, d_{n^{-}}$and $w_{n}$-subdiagrams contain no such arrows, so the set of such subdiagrams does not change.

Proof of $\Omega_{2}$-invariance: An $\Omega_{2}$-move introduces (or removes) two arrows in the Gauss diagram as in Table 2. The tails of these arrows are adjacent on the circle, and the heads are also adjacent on the circle. This is not the case for any two arrows in an $a_{n^{-}}, d_{n^{-}}$or $w_{n^{-}}$-subdiagram. There may be $a_{n^{-}}, d_{n^{-}}$or $w_{n}$-subdiagrams that contain one of the two arrows affected by the move. However, such subdiagrams come in pairs with opposite sign, since the two arrows have opposite sign and point from adjacent points on the circle to adjacent points on the circle. Hence $A_{n}, D_{n}$ and $W_{n}$ are invariant under $\Omega_{1^{-}}$and $\Omega_{2}$-moves.

Proof of invariance of $A_{n}\left(D_{n}\right)$ under descending (ascending) $\Omega_{3}$-moves: By Lemma 3 , a knot diagram invariant that is unchanged under $\Omega_{1}$ - and $\Omega_{2}$-moves is invariant under all ascending (respectively descending) $\Omega_{3}$-moves if it is invariant under moves of class $\Omega_{3--+-}\left(\Omega_{3-+++}\right)$. The corresponding moves of Gauss diagrams are depicted in Table 2.

An $\Omega_{3}$-move affects three arrows in the Gauss diagram. The move obviously does not change the number of $a_{n^{-}}$and $d_{n}$-subdiagrams that only contain one of these three arrows. The subdiagrams that contain two of the affected arrows might change. These two arrows must then have adjacent end points at some point on the circle.

Any two arrows in an $a_{n}$-subdiagram have adjacent end points at no more than one place on the circle. Then these end points are one head and one tail. If the two arrows are crossed, the tail comes before the head on the circle, and if the arrows are not crossed, the head comes before the tail. 
We see in Table 2 that for an $\Omega_{3-+++}$-move, two affected arrows that have a head and a tail next to each other are crossed if and only if the head comes before the tail. Hence no pair of arrows that are affected by an $\Omega_{3-+++}$-move can belong to an $a_{n}$-subdiagram. Consequently $A_{n}$ is invariant under descending $\Omega_{3}$-moves. In the same way we see that no pair of arrows that are affected by an $\Omega_{3--+-}$-move can belong to a $d_{n}$-subdiagram.

4.4. The Weirdness $W_{3}$. A geometric formula for the jump. The function $W_{3}$, which is given by subdiagrams on the form $w_{3}=$, is called Weirdness. We shall give a formula for the jump of $W_{3}$ under a positively directed $\Omega_{3}$-move.

Let $k$ be a knot diagram and $C$ a selected changing disk for a $\Omega_{3}$-move on $k$. The piece of knot diagram exterior to $C$ consists of three strands, which we label $a, b$ and $c$. $a$ is the strand that connects the top strand inside $C$ with the middle strand. $b$ connects the middle and the bottom strand, and $c$ connects the top and the bottom strand. By the local linking number $l l k$ of the pair $(k, C)$ we mean the following (cf. Example 3 below):

Erase the piece of knot diagram that lies inside $C$. Erase the $c$ strand. Make the remaining $a$ and $b$ strands into a two-component link diagram by connecting the two ends of each strand with a strand inside $C$ in such a way that the strand that closes the $b$ strand does not pass over the strand that closes the $a$ strand.

The local linking number is the linking number of this link. (The linking number is an invariant of two-component links. If we pick one of the components, the linking number is the sum of the signs of the crossings where this component crosses over the other.)

$l l k$ is clearly invariant under equivalence of knot diagrams (as long as we keep track of the selected changing disk.)

Proposition 5. $W_{3}$ jumps by llk $(k, C)$ under a positively directed $\Omega_{3}$-move in the changing disk $C$ on the knot diagram $k$.

Example 3 (Computing the local linking number.). Consider the changing disk $C$ in the knot diagram

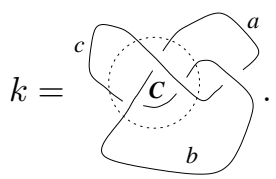

We make the knot diagram into a link diagram as below:

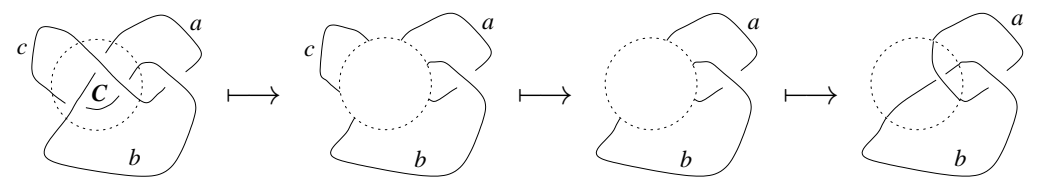

$l l k$ is the linking number of this link.

Proof of Proposition 5: By Lemma 3, we can replace an $\Omega_{3}$-move with a sequence consisting of $\Omega_{2}$-moves and a move of any $\Omega_{3}$-class with the same ascendingness/descendingness. The main trick is to make a move in the changing disk $B$ 
instead of the changing disk $A$ in the picture below:

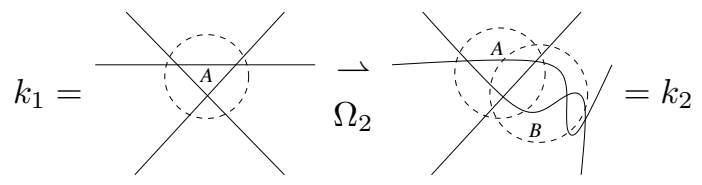

Here $l l k\left(k_{1}, A\right)=l l k\left(k_{2}, A\right)$ since $\Omega_{2}$-moves outside $A$ cannot change the linking number. $l l k\left(k_{2}, A\right)=l l k\left(k_{2}, B\right)$ since the most that can happen is that an overcrossing of the $a$ (or $b$ ) strand is replaced with an overcrossing of the $b(a)$ strand with opposite sign. This does not change the linking number. Hence it is sufficient to prove the statement for $\Omega_{3-+++}$-moves and $\Omega_{3--+-}$-moves. We consider $w_{3-}$ subdiagrams of a Gauss diagram, such that two of the arrows in the subdiagrams belong to the changing part. (Subdiagrams such that only one arrow belong to the changing part are unchanged by the move.) The changing part is depicted in Table 2. We see that there can be no subdiagrams of this kind after the positively directed move, when the changing part looks as on the right side in the Table. Before the positively directed $\Omega_{3-+++}$-move we can have $w_{3}$-subdiagrams that look like

, which contains the two dotted arrows in the changing part and one arrow pointing between unchanged segments as shown. Before the positively directed $\Omega_{3--+-}$-move we can have $w_{3}$-subdiagrams that look like

In both cases, the sign of the subdiagram is minus the sign of the third arrow in the subdiagram. The third arrow points from the segment that represents the $b$ strand to the segment that represents the $a$ strand. So when we make the move in positive direction, we add to $W_{3}$ the sum of the signs of the crossings where the $b$ strand crosses over the $a$ strand. This is the local linking number, since we close the link without introducing any new overcrossings of $b$ over $a$.

\section{Proof of Theorems 1 and 3.}

5.1. Proof of Theorem 1. We shall prove that the figure eight diagram $d_{8}$ in Figure 1 cannot be transformed into its inverse without the use of both ascending and descending $\Omega_{3}$-moves. $d_{8}$ has Gauss diagram $G_{d_{8}}=+$ while $D\left(d_{8}\right)=0$. The inverse knot diagram has $A=0$ and $D=1$ by Proposition 3 . The result follows since $A$ jumps only under ascending $\Omega_{3}$-moves and $D$ jumps only under descending $\Omega_{3}$-moves.

5.2. Proof of Theorem 3. For each knot diagram, we shall construct a knot diagram of an isotopic knot, such that the two knot diagrams can only be transformed into each other with the use of both descending and ascending $\Omega_{3}$-moves, and move of some classes $\Omega_{1 i j}$ and $\Omega_{1 k l},(i, j) \neq \pm(k, l)$. Consider the knot diagram $a$ of the unknot:

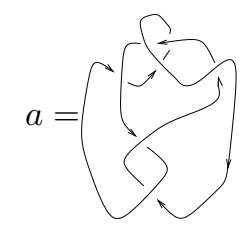


$a$ has Gauss diagrams $G_{a}=$ writhe $(a)=-1$ and winding number $(a)=0$. The inverse knot diagram $\tilde{a}$ has $D(\tilde{a})=0, A(\tilde{a})=-1$, writhe $(a)=-1$ and winding number $(a)=0$. (The writhe is unchanged under inversion, and the winding number is multiplied with -1.) For every knot diagram $d$ there is some knot diagram $k$ that is a connected sum of $d$, $a$ and $\tilde{a}$. This connected sum should be made at corresponding points on $a$ and $\tilde{a}$.

$k$ and $d$ describe isotopic knots, since we have just added two unknots. $A(k)=$ $A(d)-1$ and $D(k)=D(d)-1$ by Proposition 3, hence we need both ascending and ascending $\Omega_{3}$-moves to transform $k$ to $d$. writhe $(k)=$ writhe $(d)-2$ and winding number $(k)=$ winding number $(d)$ by Lemma 1 (because the contribution in winding number from $a$ and $\tilde{a}$ cancel). The winding number and writhe jump by $\pm i$ and $\pm j$ under an $\Omega_{1 i j}$-move and are invariant under $\Omega_{2}$ - and $\Omega_{3}$-moves, so a collection of moves of classes $\Omega_{1 i j}$ and $\Omega_{1,-i,-j}$ can never raise the writhe by 2 and leave the winding number unchanged.

\section{REFERENCES}

[1] V. Arnold, Plane Curves, Their Invariants, Perestroikas and Classifications, Preprint Forschungsinstitut für Mathematik ETH Zürich, May 1993.

[2] M. Polyak and O. Viro, Gauss Diagram Formulas for Vassiliev Invariants, Internat. Math. Res. Notices 1994 No. 11, pp. 445-453.

[3] K. Reidemeister, Elementare Begründung der Knotentheorie, Abh. Math. Sem. Univ. Hamburg 5 (1927) pp. 24-32.

[4] K. Reidemeister, Knotentheorie, Verlag von Julius Springer, Berlin 1932.

[5] B. Trace, On the Reidemeister moves of a classical knot, Proc. Amer. Math. Soc. 89 (1983), no. 4 , pp. $722-724$

Department of Mathematics, Uppsala University, Box 480, S-751 06 Uppsala, Sweden

E-mail address: olleo@math.uu.se 